\title{
Modeling Smart Agriculture using SensorML
}

\author{
Maha Arooj \\ University Of Lahore \\ Pakpattan Campus, \\ Pakistan
}

\author{
Muhammad Asif \\ University Of Lahore \\ Pakpattan Campus, \\ Pakistan
}

\author{
Syed Zeeshan Shah \\ University Of Lahore \\ Pakpattan Campus, \\ Pakistan
}

\begin{abstract}
IoT is transforming the physical world into a digital world by connecting people and things. This paper describes state of the art domains where IoT is playing a key role. Smart agriculture is selected as a case study of IoT application domain. OGC sensor web enablement framework is studied and discussed its application for smart agriculture. This paper mainly focuses on modeling the smart agriculture system using SensorML of OGC. It also identified, developed and modeled few major components/sub-systems/systems required for smart agriculture. This study also demonstrated how SensorML can be utilized in modeling IoT enabled systems.
\end{abstract}

Keywords-Internet of Things; Smart Agriculture; Sensors; SensorML; OGC SWE; Sensor Web

\section{INTRODUCTION}

Internet of Things (IoT) is an umbrella term covers many aspects of the connected world. It is enabling the objects in the real world to communicate and collaborate in everyday working or living environment. It represents the convergence of various enabling technologies such as connectivity, smart objects that can sense, store and communicate. By 2020, there will be around 50 billion physical and virtual entities connected to form a global network infrastructure[1]. Things are becoming recognizable to exchange information that must be readable, addressable and locatable through different sensing devices.

In the past few years, wireless communication proposed an innovative paradigm called the Internet of Things (IoT). It is a unique infrastructure [2] that is promptly achieving the scenario of recent wireless telecommunications. It evolve from the concepts of pervasive, ubiquitous, and ambient computing and became the most disruptive technological revolution. IoT is enabling to collect the sensed data, analyze it and perform primary actions by providing smartly intelligent management and decision making processes. This new dimension is a stepforward to develop smart socio-economic societies. In the context of domestic and working fields, IoT have obvious effects on both such as smart homes and offices, logistics, business process management, intelligent transportation of people and goods, e-health, automation or industrial built-up and enhanced learning are the examples of some application developments in which this new standard will play a prominent role in coming future [3].

Open Geospatial Organization (OGC) [4] is a standard organization that promote the development and implementation processes for geospatial services. This standard initiated Sensor Web Enablement (SWE) that constructing a distinctive and innovative structure for developing all types of Webconnected sensors systems. SensorML is an Open Geospatial
Consortium standard. It give standard models which provide a rich assemblage of metadata that make the sensor system discoverable, measureable and observable. SensorML is a sensor programming mechanism provide XML encoding for defining the sensors and its observation processes [4]. OGC also support many IoT sensor based applications and build several research projects and commercial developments into different geographical data frameworks.

In this paper, Section II describe the application domains using IoT enabling technologies. Section III introduced the SensorML based on OGC SWE framework that contain some related work where the SensorML is implemented. It also contain the description of Sensor Web and its standard elements. Accordingly, the Case study of SensorML is illustrated in section IV, which defines the working of different components of smart agriculture. Then it will conclude in Section V with a short summary of entire working as well as viewpoints on future research experiments.

\section{IOT APPLICATION DOMAINS}

This section elaborate the four different application domain areas that based on IoT paradigm.

1) Supply Chain Management: Supply chain management (SCM) is a set of coordinated activities for incorporating the dealers, producers, transporters, and consumers conveniently so that the right item or service can be delivered [5]. Global supply chain forum has identified eight main processes of SCM: consumer relationship and service management, demand management, order fulfillment, manufacturing, flow management, procurement, product development and commercialization and return [6-8]. Supply chain particularly in food (fresh or processed) require extra measures to manage the quality parameter of food items and maintain the food degradation process. Long supply chains of fresh or unpreserved foodstuff can be affected by extraordinary hazardous elements [9-13]. The Internet of Things and enabling technologies can provide solutions to manage complex processes of the supply chain. The operational efficiency in fleet management, cargo integrity monitoring, and storage condition control, optimization of warehouse workload, inventory tracking and analytics can be achieved with IoT technology. The sensing and tracking technologies such as RFID, GPS, smart labels, temperature and humidity sensors can bring in a variety of data such as location, weather conditions, traffic conditions, temperature stability and driving behaviors. Sensing, in this regard can bring deep intelligence and new business models in supply chain and logistics. 
2) Traffic Monitoring and Management: Smart traffic monitoring and management is a growing need for smart cities. With the enabling technologies, now it is becoming possible to have a smart infrastructure to control the vehicles flow on the road, provide efficient and safe road journey, accident handling, help in preventing the traffic congestion, save the travel time or cost and fuel consumption etc [14]. The objectives of smart traffic monitoring can be achieved by collecting real-time data and observation of different traffic conditions.

A number of researchers have dealt with the problem of intelligent traffic monitoring and controlling, and as a result of their efforts, several different approaches have been developed. Like one author proposed a solution to improve the precise automobile location and get the mechanical information of vehicle status by the technology of wireless data communication and RFID technology [15]. In another article, the authors developed strategies to integrate different dynamic data into Intelligent Transportation Systems by using Wireless Sensor Networks [16].

The Author [17] proposed a design for intelligent traffic monitoring system (ITMS) by implementation of the Internet of Things (IoT) that visualize the traffic on the Web-based GPS or GPRS. This IoT implementation focused on three components such as the attainment of traffic that contain the abilities of GPS sensor, GPRS-based data transport, and the scheme of a Web/GIS-based traffic monitoring software. Patrik et al. [18] proposed a service-oriented architecture (SOA) for an effective integration of IoT in enterprise services. Recently researchers shifted their attention to revolutionizing paradigm of the Internet of Things, which resulted in constructing of a more convenient environment composed of various intelligent systems in different domains.

3) Waste Management System: The Intelligent waste management system will provide beneficial information to the public by helping and encouraging a useful and optimizing method of collecting waste. It comprises of different processes like the collection, manage and checking the waste things, transportation, handling of garbage data and the clearance of items. Whole processing of this system is done on a cloud platform that supports sensing as a service. Commercial Waste management is divided into some selective methods such as the gathering, transportation, discarding, handling, controlling and monitoring of waste ingredients. According to this scenario, different sectors are involved such as health and safety authorities, reusing and manufacturing industries and city governance. All these sector processes retrieve information according to their own concern.

4) Smart Agriculture: Agriculture is an essential part of world's economy and facilitates many business entities and communities around the world. According to World Economic Forum [19], the demand for a feed of 9 billion world population will rise to 70 percent by 2050 . To meet this challenge, improvements in the global food system is required that can meet the requirements of farmers as well as consumers. Grow Africa initiative [20] is a kind of joint venture by World Economic Forum and African Union Commission(AUC) to target collaboration among key stakeholders and improve agricultural growth. To achieve the objective of growth rate by 20 percent requires a transformation of the agriculture sector by increasing the collaboration by all stakeholders including

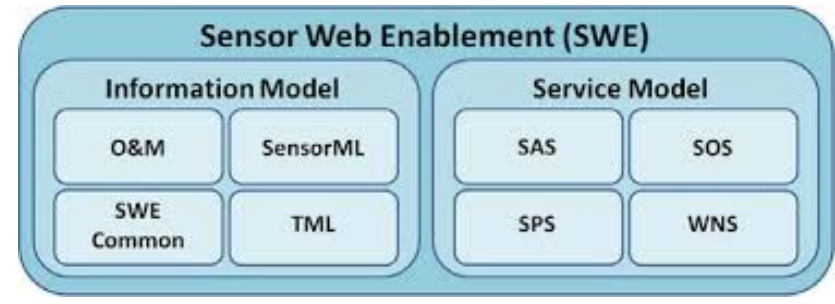

Fig. 1. Overview of the Sensor Web Enablement architecture [27].

farmers, marketers, government, civil society, and the private sector. Smart agriculture systems can pave the way to achieve the global objectives of food security and agriculture. Smart agriculture system mostly defined in five factors: environmental, social, economic, geographical and technical aspects. Smart agriculture systems have the potential in provisioning in agricultural management, production and overall distribution processes [21, 22].

The enabling technologies in IoT are going to modernize agriculture around the world particularly in developed countries. The smart agriculture systems are widely being used in crop breeding, preservation of crops, forestry and insect operation, and agricultural meteorological conditions[23, 24]. Sensing capabilities and platforms are required to monitor the plant growth status, soil conditions, pest controlling, and weather conditions.

\section{SENSOR WeB}

This section presents Sensor Web architecture that derive from Open Geospatial Consortium (OGC) Sensor Web Enablement including its other information and service models. Open Geospatial Consortium (OGC) is an international standardization organization comprises of various services that integrate sensors and sensor data into geographical data frameworks. SWE define the designs for sensor data and metadata along with sensor service interfaces. Collectively, these standards create a structure that achieving the objectives of the Sensor Web. It comprises of different standard elements:

- Observations and Measurements $(O \& M)$ is an encoding for data being observed or measured by the devices.

- Transducer Markup Language (TML) is augmented for data streaming and supports the encoding of sensor metadata or data. Though, it also addresses various application area than O\& M and SensorML.

- The Sensor Observation Service (SOS) describes the network-centric data demonstrations and processes for retrieving and incorporating the observation data from sensor systems

- The Sensor Planning Service (SPS)setting the measurement parameters and used for tasking sensors.

- The Sensor Alert Service (SAS) describes the service interface which can be used by the user for contributing to self-defined alert situations. This service provides the facility of receiving notification in case the circumstances are matched 
- Web Notification Service (WNS) identifies an interface for a service avail by the user for message interchange with multiple services.

1) SensorML: The SensorML specification [25, 28] describes the models, encodings and XML Schema for any activity, counting measurement by a sensor system. SensorML is a process model that cover the specifications, input/output, metadata quality, filters, procedures, standardization data, several probable parameters and useful characteristics that can apply in SensorML descriptions. Different subtypes clarify different varieties of sensors, actuators or structures of devices. Global Positioning System (GPS) sensor is an example that also integrates the system of complex processes that gives the observations of location, direction, and speed.

SensorML provides an operational model of the sensor system, rather than a thorough depiction of its hardware. It uses sensor structures and a systems modules as processes such as actuators, devices, detectors, platforms, etc. Therefore, each element can be involved in more than one processes that provide a facility for geolocating and handling or define the observations to a higher level of information. In SensorML, all methods containing the sensors and sensor systems have input/output, constraints, and processes that can be operated by applications for developing measurements from any sensor system. Furthermore, SensorML also gives other metadata that is beneficial for empowering the discovery process, finding system constraint of security or authorized usage for giving connections and references, physical properties and explaining task-able properties.

SensorML and Sensor Web Enablement processes are the key discovery in the development of detector applications. Sensor Model Language (SensorML) includes the sensors and actuators that give descriptions of sensors and sensor systems for record management. SensorML process the information system and observation discovery to support the geolocation of measured values. Its services supported the sensor discovery, sensor programming mechanism, sensor geolocation, and contribution to sensor alerts and processing of sensor observation [27] [28].

A sensor has only one input and output term for both the scalar quantities. According to SensorML, a sensor describes a specific type of Process Model. It clarifies the element that the SensorML can be used to make the immediate structure of a System. This system measures the temperature, pressure, wind speed, wind direction, and rainfall amount. All these detectors using the five different modules for observation and measurement such as a thermometer for temperature detection, a barometer for measuring wind direction, wind sensor, rain gauge and spark fun for soil moisture detection.

\section{Smart Agriculture}

By considering the working of SensorML, several Authors presented their work implementing its processes. The paper [29] presented the system architecture which is stimulated by the Open Geospatial Consortium (OGC) Sensor Web Enablement (SWE) based on one of its information model. It supported Sensor Model Language (SensorML) of which Process Model is primary for efficiently handling the heterogeneous devices and their information. Author designed prototype by using "SensorModel V1.0" and executed used to create the standard model for integrated management of various remote sensing satellite sensor resources information and determine the model-based recovery and conception of connected remote instruments and their data. It stimulates the inclusive retrieving and collective formation and monitoring the accessible distant sensors information in time-critical hazard emergency.

Aloisio G et al[28], proposed a Globus Monitoring and Discovery Service that integrate sensor networks in Grid environments by means of the design of an information system based on Sensor Modeling Language (SensorML). It is a method that based on Monitoring and Discovery Service of Globus Toolkit to assimilate devices in grid environments. These grid sensors provide explicit information about various phenomena and utilizing the computing resources in a resourceful and coordinated manner.

SensorML, however, provide efficient mechanism for describing sensor resources as well as Scientific Workflow. Scientific Workflows usually works for modelling and executing scientific experiments. Its technology helps the researchers by letting them to capture in a machine executable manner to the process concerning to some research. SensorML in this, facilitate as a tool by giving some extensions for distributed Scientific Workflows Description on the Web [29].

\section{Case Study of Smart Agriculture}

In this section, the proposed simulation mechanism is introduced. The user can access the sensor data, by using web application named The Smart Miner. User can create his account and avail the service. All these sensor specifications stored in registry, can select data from the search panel and select required detector information. System can process these devices behavior and generate information by considering observation and measurement element. Data source interface contains the sensor id, source name and source table. The user can also add new data source item in it.

In these schemas, five system components are elaborated that describe different functionality according to their specification.

1) Temperature Detector: Temperature sensor detector used to detect humidity level in the environment. It contains the sensor name, type, and identification numbers, temporal, reference to the platform description, sensor's location, the sensor operator and tasking services, response characteristics and information for geolocating measures, textual metadata, and history of the sensor and classification constraints of the description. In Identification component of temperature sensor, a name and a model number are given for the detector. Each Term is clearly stated by the URN. The calibration curve in temperature detector gives representing of input values to the output values at a stable state system. There is a separate virtual input for every measured development. In output term, all values and a time tag is measured.

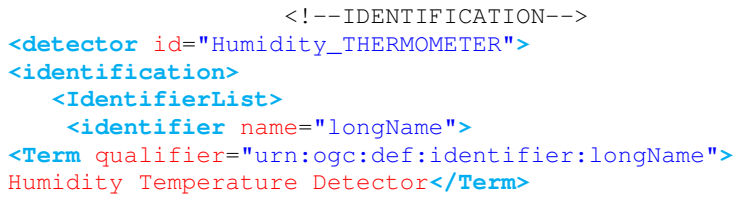




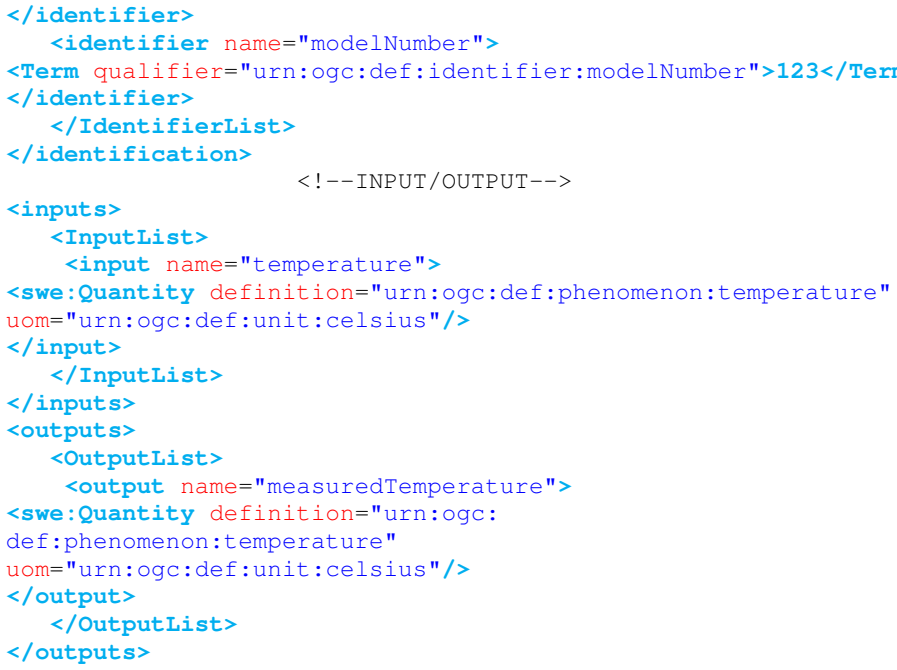

Listing 1. SensorML description of Temperature Detector

2) Wind Speed Detector: Anemometer sensor or wind speed detector is used to detect the wind speed that measures the air velocity and air flow. In identification component of wind speed detector, a unique identification and model number needs to give and its each Term is defined by URN. The calibration curve in Wind Speed sensor, representing of input values to the output values at a stable state system. There is a separate virtual input for every measured development. In output term, all values and a time tag is measured.

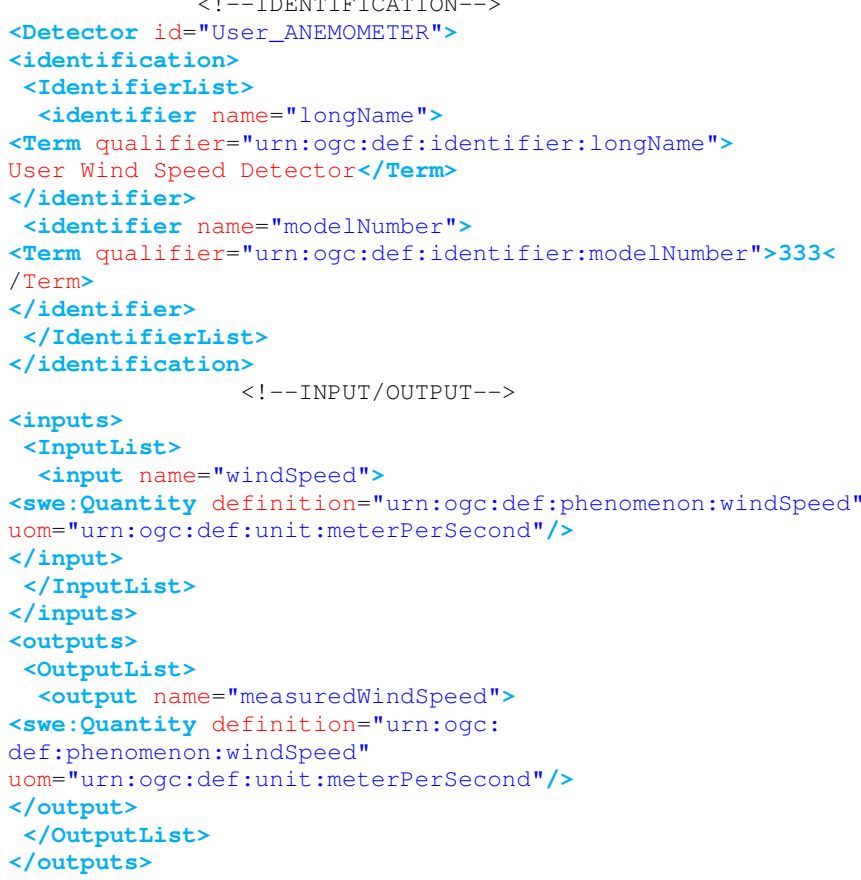

Listing 2. SensorML description of Wind Speed Detector

3) Wind Direction Detector: Wind direction detector also has a unique name and a model number as its each Term is clearly stated by the URN. When specifying the position, id will be used. The user can read this measurement data by different parameters, input or output conditions and calibration time according to it. Different rotation of axis observe the status of the direction of the wind and give information to the user. In identification component of wind direction detector, a unique identification and model number needs to give and its each Term is defined by URN. The calibration curve in Wind direction sensor, representing of input values to the output values at a stable state system. There is a separate virtual input for every measured development. In output term, all values and a time tag is measured.

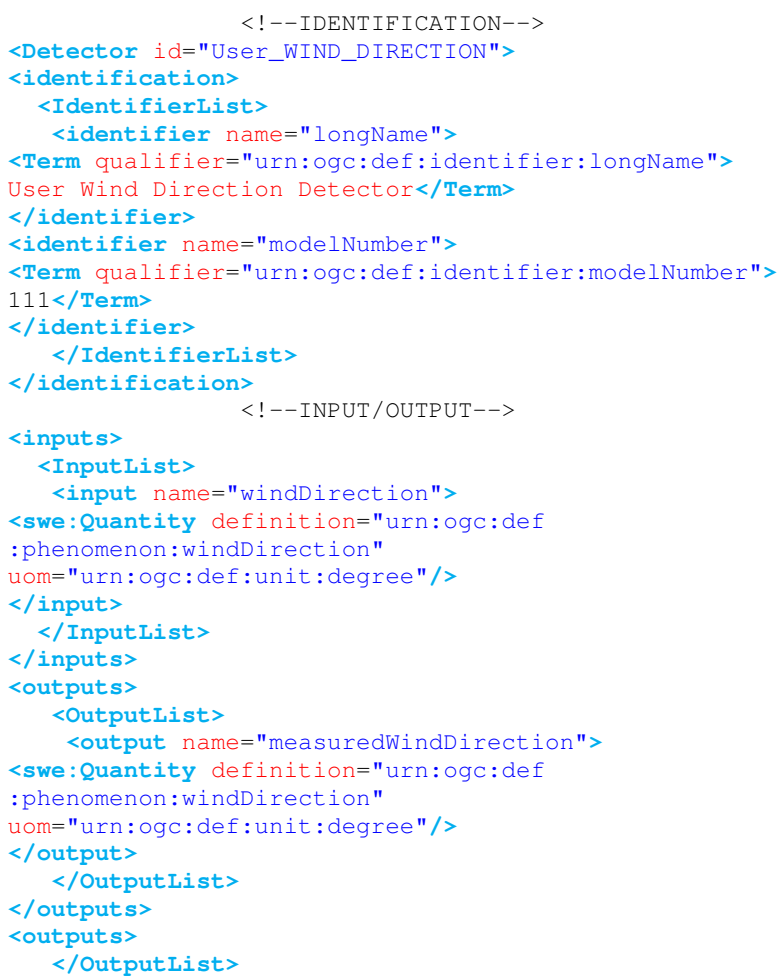

Listing 3. SensorML description of Wind Direction Detector

4) Rainfall Detector: Rain gauge sensor instrument is used for rainfall measurement. Like others, this sensor also has identifier name and model number. Reference frame contains all information of the rainfall gauge. Its origin is situated at the connector or case junction. The parameter includes the calibration time, rainfall measurement, quantity and gives steady state response condition of the detector. In identification component of Rainfall detector, a unique identification and model number needs to give and its each Term is defined by URN. The calibration curve in rainfall sensor, representing of input values to the output values at a stable state system. There is a separate virtual input for every measured development. In output term, all values and a time tag is measured.

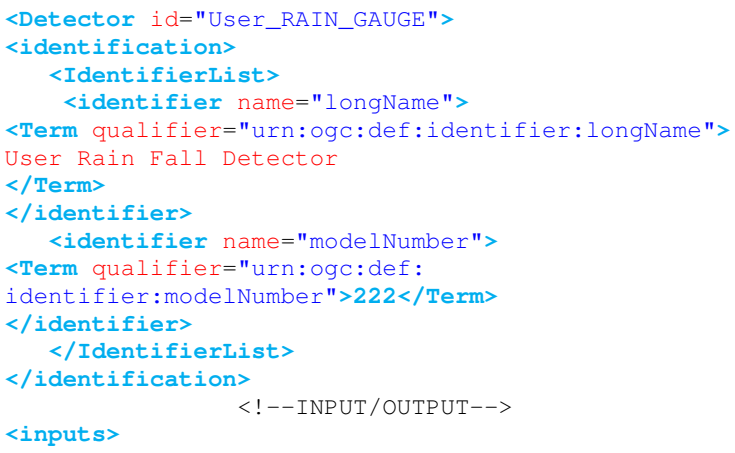




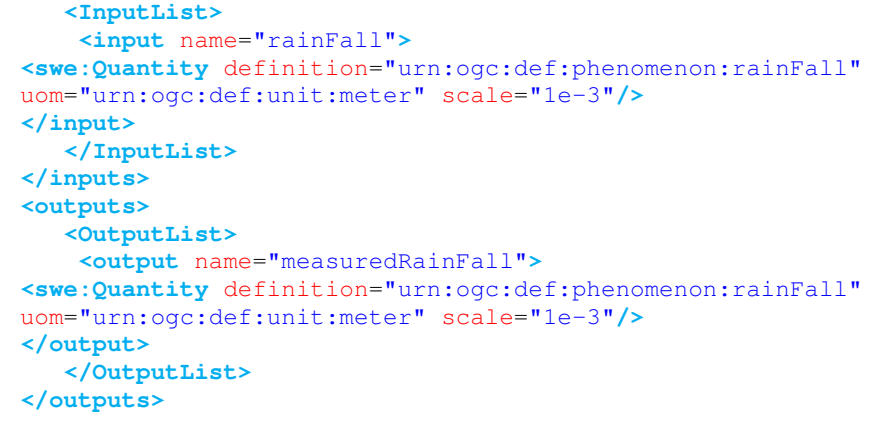

Listing 4. SensorML description of Rainfall Detector

5) Soil Detector: Soil Detector measure the soil moisture on land. Although others, it also has identification name and model number. It measures the water potential or checks the volumetric content of water in a specific land area. Such useful information helps the user in making an appropriate decision about the crop. In identification component of soil detector, a unique identification and model number needs to give and its each Term is defined by URN. The calibration curve in soil sensor, representing of input values to the output values at a stable state system. There is a separate virtual input for every measured development. In output term, all values and a time tag is measured.

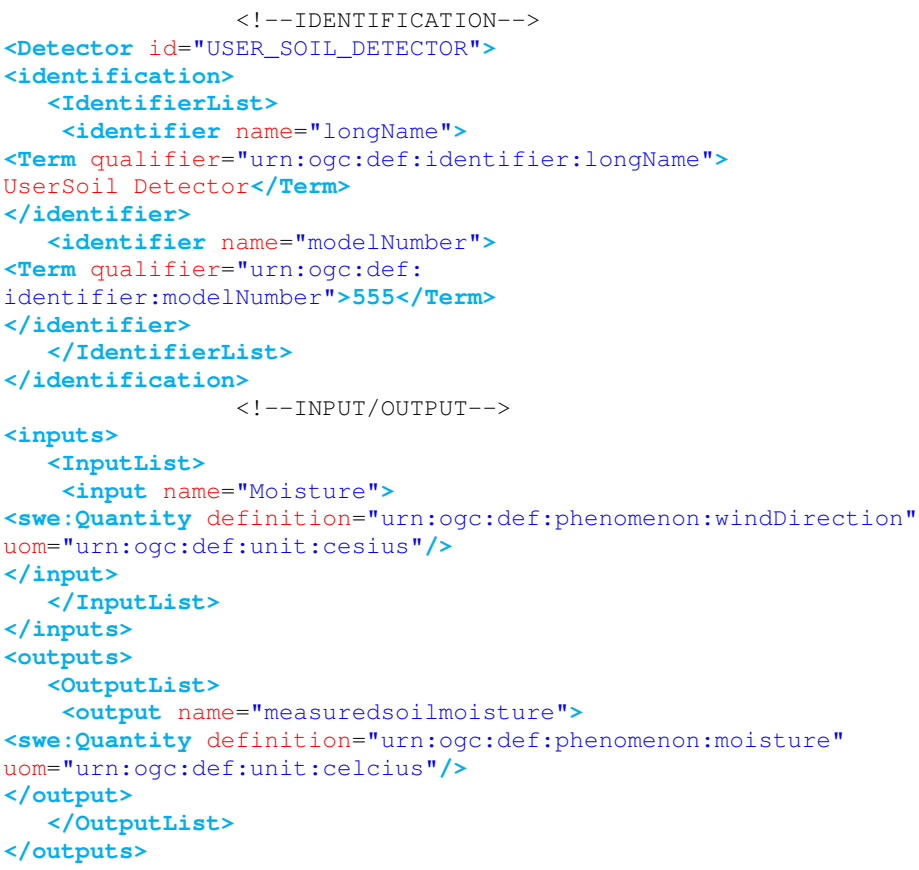

Listing 5. SensorML description of Soil Detector

\section{CONClusion}

This paper provides an overview of the application domains of IoT. Particularly, smart agriculture as a case study has been taken to model using SensorML. Different components/subsystems/systems of smart agriculture have been identified and their SensorML based model has been presented. The applicability of SensorML to model IoT based systems is evaluated. The descriptive capability of SensorML can help to describe and model IoT based systems. In future work, a wide scale modeling of smart agriculture will be performed to evaluate the descriptive capabilities of the SensorML.

\section{REFERENCES}

[1] Ericsson, More than 50 billion connected devices, White Paper, February 2011, Online: http://www.ericsson.com/res/docs/whitepapers/wp-50billions.pdf

[2] Reed, D.A., D.B. Gannon, and J.R. Larus, Imagining the future: Thoughts on computing,Computer, 2011(1): p. 25-30

[3] Atzori, L., A. Iera, and G. Morabito, The internet of things: A survey, Computer networks, 2010. 54(15): p. 2787-2805.

[4] Botts, M. and A. Robin, OpenGIS sensor model language (SensorML) implementation specification OpenGIS Implementation Specification OGC, 2007. 7(000).

[5] Xu, L.D., Information architecture for supply chain quality management., International Journal of Production Research, 2011. 49(1): p. 183-198.

[6] Melo, M.T., S. Nickel, and F. Saldanha-da-Gama, Facility location and supply chain managementA review. , European journal of operational research, 2009. 196(2): p. 401-412.

[7] Liu, J., Zhang, S., and Hu, J. (2005). A case study of an inter-enterprise workflow-supported supply chain management system. Information and Management, 42(3), 441-454

[8] Liu, J., S. Zhang, and J. Hu A case study of an inter-enterprise workflowsupported supply chain management system. The International Journal of Logistics Management, 2001. 12(2): p. 13-36.

[9] Van der Vorst, J.G., S.-O. Tromp, and D.-J.v.d. Zee, Simulation modelling for food supply chain redesign; integrated decision making on product quality, sustainability and logistics. International Journal of Production Research, 2009. 47(23): p. 6611-6631.

[10] Xiaorong, Z., et al., The Design of the Internet of Things Solution for Food Supply Chain. 2015

[11] Kelepouris, T., K. Pramatari, and G. Doukidis RFID-enabled traceability in the food supply chain. Industrial Management and Data Systems 2007. 107(2): p. 183-200.

[12] Barchetti, U., et al. RFID, EPC and B2B convergence towards an item-level traceability in the pharmaceutical supply chain. in RFIDTechnology and Applications (RFID-TA), 2010 IEEE International Conference on. 2010. IEEE.

[13] Yu, X., et al., Pharmaceutical supply chain in China: current issues and implications for health system reform Health Policy, 2010. 97(1): p. 8-15.

[14] Yu, X., F. Sun, and X. Cheng. Intelligent urban traffic management system based on cloud computing and Internet of Things. in Computer Science and Service System (CSSS) 2012 International Conference on. 2012. IEEE

[15] Rahman, T.A. and S.K.A. Rahim. RFID vehicle plate number (e-plate) for tracking and management system. in Parallel and Distributed Systems (ICPADS) 2013 International Conference on. 2013. IEEE.

[16] Katiyar, V., P. Kumar, and N. Chand, An intelligent transportation systems architecture using wireless sensor networks. International Journal of Computer Applications, 2011. 14(2): p. 22-26.

[17] Widyantara, I.M.O. and N.P. Sastra. Internet of Things for Intelligent Traffic Monitoring System: A Case Study in Denpasar computing, 2015. 2: p. 3.

[18] Spiess, P., et al. SOA-based integration of the internet of things in enterprise services. in Web Services, 2009. ICWS 2009. IEEE International Conference on. 2009. IEEE.

[19] Forum, W.E., Global Challenge on Food Secuity and Agriculture. 2016

[20] Africa, G. Grow Africa Initiative. . 2016

[21] Hu, S., et al., AgOnt: Ontology for Agriculture Internet of Things, in Computer and Computing Technologies in Agriculture IV. 2011 Springer. p. 131-137.

[22] Yan-e, D Design of intelligent agriculture management information system based on IoT. in Intelligent Computation Technology and Automation (ICICTA) 2011 International Conference on. 2011. IEEE.

[23] TongKe, F., Smart Agriculture Based on Cloud Computing and IoT. Journal of Convergence Information Technology, 2013. 8(2).

[24] Liang, Y., et al., Study on the framework system of digital agriculture Chinese Geographical Science, 2003. 13(1): p. 15-19.

[25] Botts, M., Sensor Model Language (SensorML) Implementation Specification, Version 1.0. OGC document, 2007. 7(000). 
[26] Lance McKee, M.B., A Sensor Model Language: Moving Sensor Data onto the Internet. April 1, 2003.

[27] $\mathrm{Hu}, \mathrm{C} ., \mathrm{N}$. Chen, and C. Wang. Remote sensing satellite sensor information retrieval and visualization based on SensorML. in Geoscience and Remote Sensing Symposium (IGARSS), 2011 IEEE International. 2011. IEEE.
[28] Aloisio, G., et al. Globus monitoring and discovery service and sensorML for grid sensor networks. in Enabling Technologies: Infrastructure for Collaborative Enterprises, 2006. WETICE'06. 15th IEEE International Workshops on. 2006. IEEE.

[29] Van Zyl, T. and A. Vahed. Using sensorml to describe scientific workflows in distributed Web Service environments. in Geoscience and Remote Sensing Symposium, 2009 IEEE International, IGARSS 2009. 2009. IEEE. 